\title{
DESIGN AND SYNTHESIS OF COORDINATION COMPOUNDS WITH TETRADENTATE LIGANDS DERIVED FROM ALKYLATED THIOSEMICARBAZIDE
}

\author{
M.Cocu*1, J. Gradinaruํ, M. Revenco ${ }^{2}$, E. Rybak-Akimova ${ }^{3}$, N. Gărbălău \\ ${ }^{1}$ Institute of Chemistry of Academy of Sciences of Moldova, Academiei str. 3, MD-2028 Chisinau, Republic of Moldova \\ ${ }^{2}$ State University of Moldova, Mateevici str. 60, MD-2028 Chisinau, Republic of Moldova \\ ${ }^{3}$ Tufts University, Talbot Ave 62, Medford, MA 02155, USA \\ E-mail:mariacocu@gmail.com; Phone: 37322 739963,Fax: 37322739954
}

\begin{abstract}
This work is devoted to the investigation of the template reactions of organic compounds derived from S-alkylisothiosemicarbazide and $\beta$-dicarbonylic compounds (acetyl(benzoyl)acetone, 2,6-diformylpyridine, 2,6-diformyl-4-methylphenol, 1-hydroxy-2-naphthaldehyde, sodium nitromalonic dialdehyde, 1,1,3,3-tetraetoxypropane, 3-ethoxyacroleine) in the presence of 3d-metal ions, determination of the structure and properties of the prepared complexes.

There were elaborated original methods for synthesis of open-chain or macrocyclic coordination compounds of nickel(II), copper(II), oxovanadium(IV) and cobalt(II) with $\mathrm{N}_{4}, \mathrm{~N}_{3} \mathrm{O}$ and $\mathrm{N}_{2} \mathrm{O}_{2}$ tetradentate ligands. Two types of hexaazamacrocyclic systems with trans- and cis- arrangement of thiosemicarbazide fragments have been obtained using template condensation of different precursors.

The biostimulative properties (biomass accumulation and increasing of enzymatic activity) of complexes have been established. Some complexes of nickel(II) have been studied as coloring agents for plastics.
\end{abstract}

Keywords: Template synthesis; coordination compounds; 3d-metal complexes; Schiff base; S-alkylisothiosemicarbazide.

\section{Introduction}

For some time, semi- and thiosemicarbazones have been a subject of interest to researchers of different profiles. In view of the fact that these organic compounds form with many metals complexes of diverse chemical, physical and structural characteristics, they are of special interest for the specialists in coordination chemistry [1-5]. In addition, many of these organic reagents as well as their metal complexes, have shown a wide spectrum of biological activity, so that they have also become a subject of intense research for pharmacologists [6].

A number of complexes, primarily of transition metals, involving thiosemicarbazones of different denticity have been prepared and investigated [1-8]. In contrast to thiosemicarbazide fragment, which ones are usualy coordinated via azometinic nitrogene and sulfur atoms, the isothiosemicarbazides and their derivatives, as a rule, involve for coordination the nitrogen atom of the isothioamide group instead of the sulfur [3,9].

A consequence of this fundamental difference in the coordination mode of thiosemicarbazones and isothiosemicarbazones is the change in the reactivity of the $\mathrm{NH}_{2}$-group. Hence, in the case of isothiosemicarbazones, this group undergoes condensation reaction with bifunctional carbonyl compounds, yielding, among others, macrocyclic complexes $[4,5]$.

\section{Results and discussion}

The main goal of this work is the design and synthesis by template method of new coordination compounds with diverse composition, on the matrix of different metals using S-alkylisothiosemicarbazides and different carbonylic compounds as ligsons.

As dicarbonylic compounds were used acetylacetone, benzoylacetone, nitromalonic dialdehyde, 3-ethoxyacroleine, 1,1,3,3-tetraetoxypropane, 1-hydroxy-2-naphthaldehyde, 2,6-diformylpyridine, and 2,6-diformyl4-methylphenol.

The study of the reaction of S-methylthiosemicarbazide with acetylacetone in the presence of oxovanadium(IV) and cobalt(II) ions showed that the property to orientate the reactant species is characteristic not only for nickel( II) ions, but for other metal ions too. In both cases the formation of the compounds $\mathbf{1}$ and $\mathbf{2}$, with open-chain ligand acetylacetone bis-(S-methylthiosemicarbazone) according to the scheme 1 was observed:

The acetylacetone bis-(S-methylthiosemicarbazone) in solutions is involved in a series of tautomeric and protolitic transformations (Scheme 2), and upon the value of $\mathrm{pH}$ and the nature of metal ions, different forms can be stabilized. For the nickel and cobalt(II) cases the moderate alkalinity (presence of acetate ions) gives rise to the stabilisation in the complex 2 of the uninegatively charged tetradentate $\mathrm{N}_{4}$ ligand, (form F). When the reaction occurs in the presence of 
oxovanadium (IV) ions, the formation of the complex 1 requests a more alkaline medium (the presence of a strong base triethylamine), and as a result, the stabilisation of the twice deprotonated $\mathrm{N}_{4}$ ligand (form $\mathrm{E}$ ) is observed.

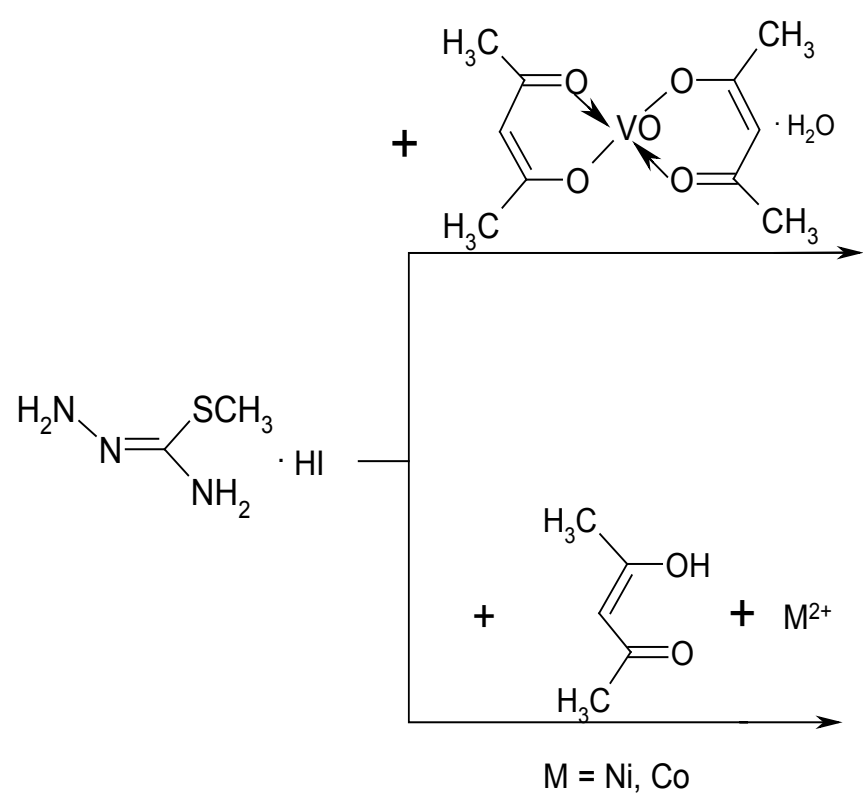

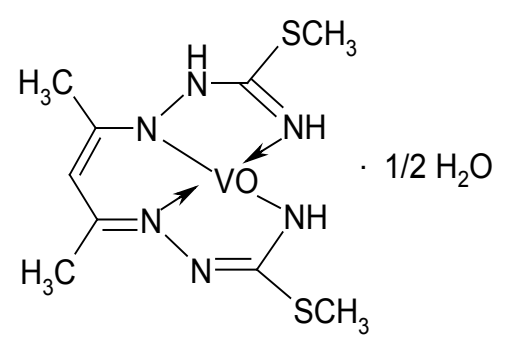

$\mathrm{VO}\left[\mathrm{HL}^{1}\right] \cdot 1 / 2 \mathrm{H}_{2} \mathrm{O}(1)$

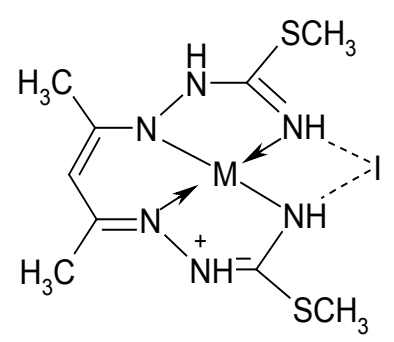

$\mathrm{Co}\left[\mathrm{HL}^{1}\right] \cdot \cdot \mathrm{H}_{2} \mathrm{O}(\mathbf{2})$

Scheme 1<smiles>C/C(C/C(C)=N/N=C(/N)[Si])=N/N=C(\N)[SiH3]</smiles>

(A)<smiles>[C]#C</smiles><smiles>CC(=N)NN/C(C)=C\C(C)=N/N=C(\N)[Se]</smiles>

(C)
$\mathrm{SCH}_{3}$

(B)<smiles>C/C(=C/C(C)=N\N=C(/N)[Si])N/N=C(/N)[Si]</smiles>

$\uparrow$<smiles>CC(=N)NN/C(C)=C\C(C)=N/NC(=N)S</smiles>

(D)<smiles>[AlH2]</smiles><smiles>CC(=N)NN/N=C(/C)C=C(C)C(=N)S</smiles>

(F)<smiles>C/C(=C/C(C)=N/N=C(N)N)NNC(=N)[SiH3]</smiles>

(E)

Scheme 2 
The nature of the initial $\beta$-dicarbonilic precursor il also responsible for the sense of the reactions in these systems. In the case of benzoylacetone the keto-enole tautomerism is much more directed to the formation of the enolic species and only the formation of benzoylacetone mono-S-alkylisothiosemicarbazone is possible. This organic reagent can be involved in a lot of template reactions. In the presence of nickel ions the formation of two types of complexes (3 and 4) has been observed (Scheme 3). After separation, purification of the products an X-ray investigation has carried out. The molecular structures of the complexes are presented in the Figs. 1 and 2.

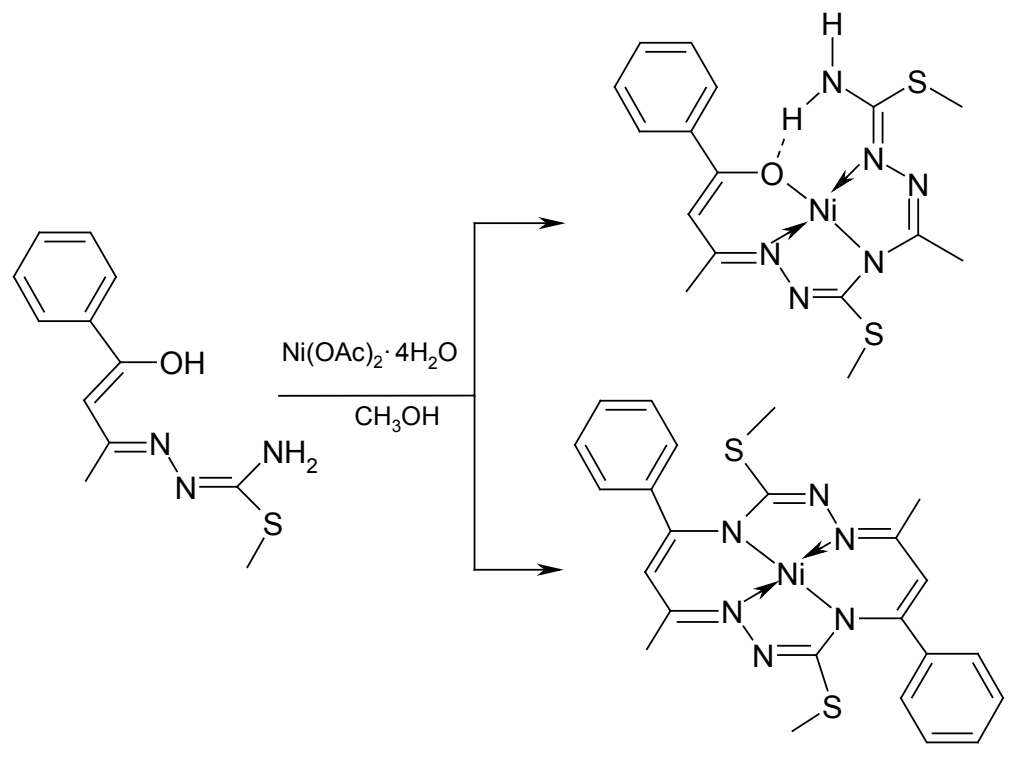

$\left[\mathrm{NiL}^{2}\right](3)$

Scheme 3

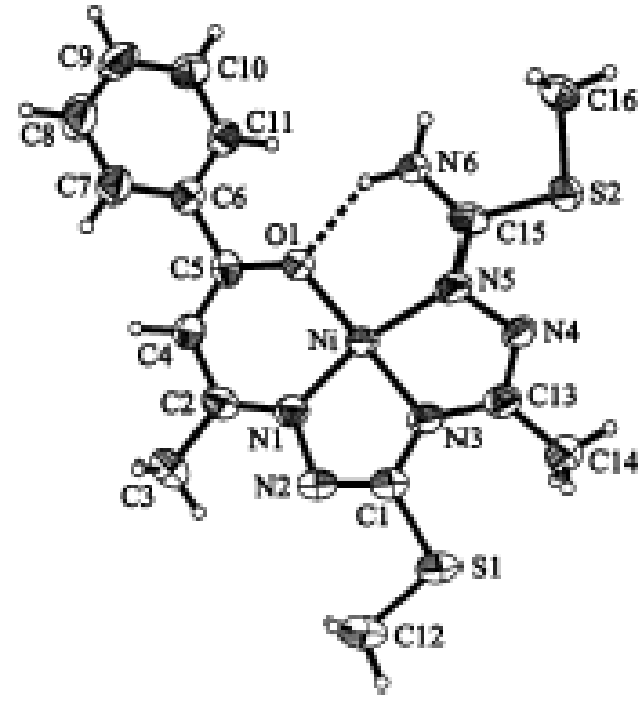

$\left[\mathrm{NiL}^{2}\right](3)$

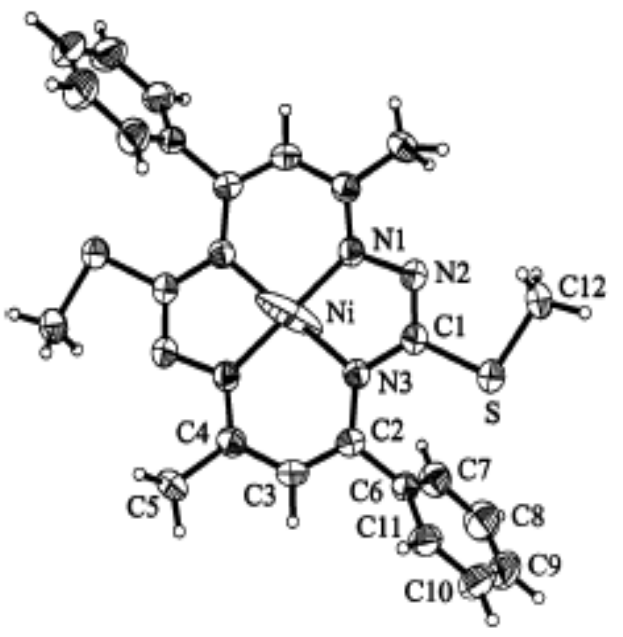

$\left[\mathrm{NiL}^{3}\right](4)$

Fig. 1. Molecular structure of the complexes $\mathbf{3}$ and $\mathbf{4}$

The appearance of $\mathrm{NiL}^{3}(4)$ complex is an usual already process, which can be explained by the condensation of two molecules of benzoylacetone mono-(S-methyllthiosemicarbazone) in the coordination sphere of nickel ion [11]. The synthesis of $\mathrm{NiL}^{2}(3)$ denotes a much more complicated state of the system, which is attended with a degradation of the initially compound with elimination of acetophenone. This compound may be considered as pseudo-macrocyclic, stabilized through the formation of hydrogen bond. This structure, once more, shows the possibility of acyclic arrangement of the systems around the metal ion, facilitated by the formation of the hydrogen bonds of marginal group. 
An unexpected result is the coordination mode of the isothiosemicarbazidic fragment in $\left[\mathrm{NiL}^{2}\right]$. Coordination of the second fragment via $\mathrm{N}(5)$ atom is dictated by the stereochemical peculiarities of the nickel ion, which one has the tendency to form compounds with square- planar configuration and by versatile character of the thiosemicarbazide fragment's coordination.

This phenomenon is emphasizing, when the number of coordination centres offered by ligand exceeds the requirements of central atom. For optimization of the structure and to satisfy the stereochemical necessity of the central ion the ligand accepts an unusual conformation. This behaviour was also observed in the case of nickel coordination compounds with 2,6-diformyl- and 2,6-diacetyl pyridine bis-(S-methylthiosemicarbazone) [10]. In general these two ligands are potentialy pentadentate and can be coordinated by the metal ions through $\mathrm{N}_{5}$ set of donor atoms, including the pyridine nitrogen, two azometinic nitrogen atoms of the both isothiosemicarbazidic moieties, as well as the marginal nitrogen atoms of the same fragments. This coordination mode was confirmed for the manganese 2,6-diacetylpyridine bis-(S-methylthiosemicarbazone) compound, where the organic ligand is pentacoordinated by the central atom in the equatorial plan. In the case of nickel complexes the both ligands adopt a tetradentate coordination as is shown in the Fig. 2 for the 2,6-diformyl-derivative.

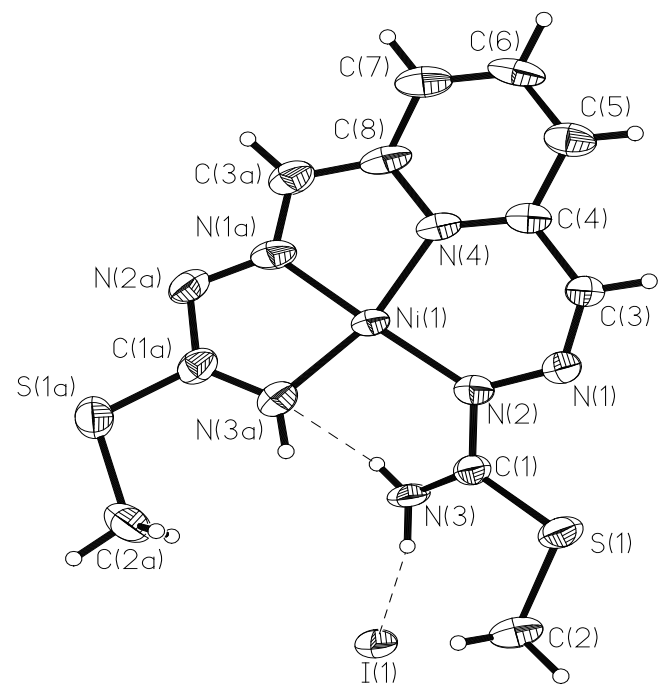

$\left[\mathrm{Ni}\left(\mathrm{HL}^{4}\right)\right] \mathrm{I}(\mathbf{5})$

Fig. 2. Molecular structure of the complex 5

The presence of the double bonds in the organic reagent invokes an effective conjugation on whole molecule, which prompts a planar structure. These desiderates offered by ligand are not accepted by nickel ions, which dictates to the ligand an other configuration. The both isothiosemicarbazidic fragments are condensed in different configurations, vis-à-vis to azometinic bonds $-\mathrm{RC}=\mathrm{N}$-. One fragment adopts $\mathrm{E}$ configuration and uses the marginal nitrogen atoms for coordination, an the second one - a $\mathrm{Z}$ configuration, which offers the possibility to be coordinated to the nickel ion via donor atom N(2). Thus, nickel ion conducts the reaction of condensation in a certain way and hinders the formation of compound, which can contain both thiosemicarbazidic fragments in E configuration. This is an example of metal promoted reactions. For the same central atom the nature of the dicarbonylic block has an essential impact on the complex geometry. Comparing the nickel compounds prepared by template reaction of S-methylthiosemicarbazide with 2,6-diformyl- or 2,6-diacetyl-pyridine, the formation of the complexes of the same composition NiLI can be observed. The difference is manifested in the complex configuration: one of them has a plan-square structure, the second - the configuration of a tetragonal pyramid with coordination of the iodine ion in apical position.

Apparently there are no significant changes of the power of the crystalline field formed by ligand after substitution of the hydrogen in 2,6-diformylpyridinic moiety by methyl group. The observed change of complex geometry can be explained by the presence of the methyl bulk groups which ones taking off the species [NiL] ${ }^{+}$ one of another, creates conditions to arrange the iodine ion in apical position, so the coordination number of nickel becomes 5 . In the case of 2,6-diformilpyridine, these restrictions are far-off, the species $[\mathrm{NiL}]^{+}$are arranged more compact, so in apical position is placed the sulfur atom of the neighboring molecule. At these conditions, distance $\mathrm{Ni}$-S remains less than the sum of the Van der Waals rays and can not be consider as a chemical bond. The complex configuration remains square-planar. 
Combination of the benzoylacetone mono-S-alkylisothiosemicarbazone with other carbonylic components (for example 2, 6-diformyl-4-methylphenol or 2-oxynaphthaldehyde) makes possible to obtain on the matrix of nickel, cobalt, oxovanadium(IV) or copper(II) new coordination compounds containing double condensed isothiosemicarbazide fragment [12]

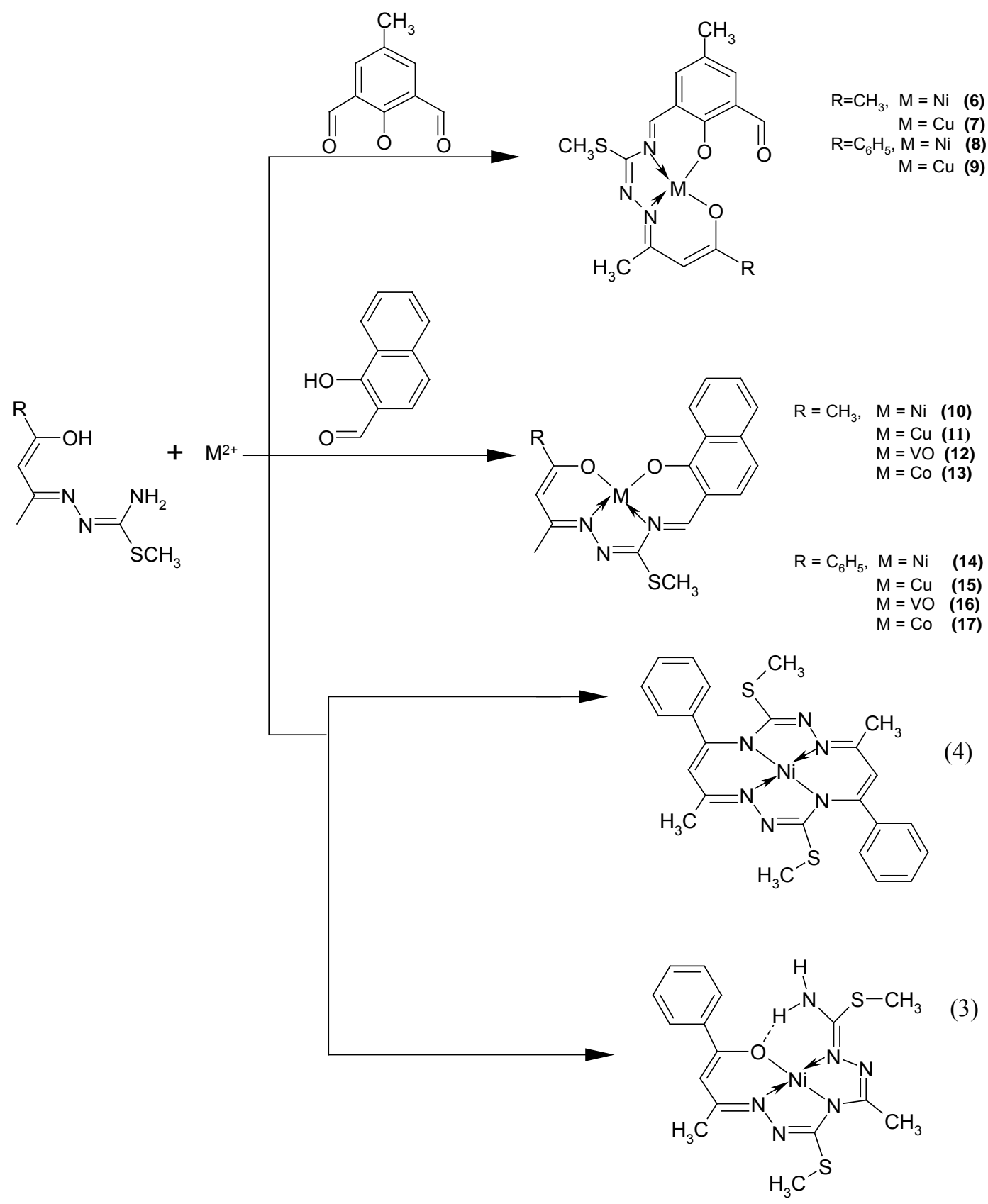

Scheme 4

The formation of the complexes 6-17 has been demonstrated using a number of spectral, magnetic, and chromatographic methods. For the complexes $\mathbf{1 0}$ and $\mathbf{1 3}$ an X-ray investigation has been undertaken. Their molecular structures are presented in the fig. 3 .

The above mentioned complexes 6-17 are not the only species, which are present in the system. The partial desegregation of the initial ligson produces fragments with an increased reactivity, which ensures their fit in new combinations, with an unusual arrangement of atoms and bonds. In the presence of 2,6-diformyl-4-methylphenol or oxynaphthaldehyde the formation of the complexes $\mathbf{3}$ and $\mathbf{4}$ has been also observed. These transformations prove the complexity of the process which induces the diversity of the final products. Thus, the reaction with the higher yield can not be considered as single possible transformation. It is the reaction the more advanced, but not the only process. 
The lockout of another process as frequently occurs by solvent implication. The interaction of benzoylacetone monoS-methylthiosemicarbazone with 2,6-diformyl-4-methylphenol in methanol gives rise to the condensation in molar ratio $1: 1$. The acetals formation prevents the condensation with the second group and thus the generation of dinuclear compound [12].

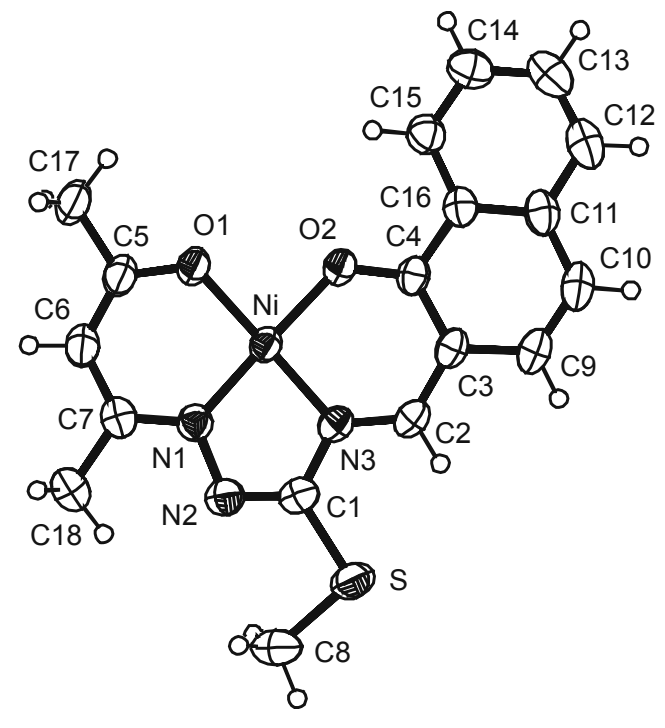

$\left[\mathrm{NiL}^{7}\right](\mathbf{1 0})$

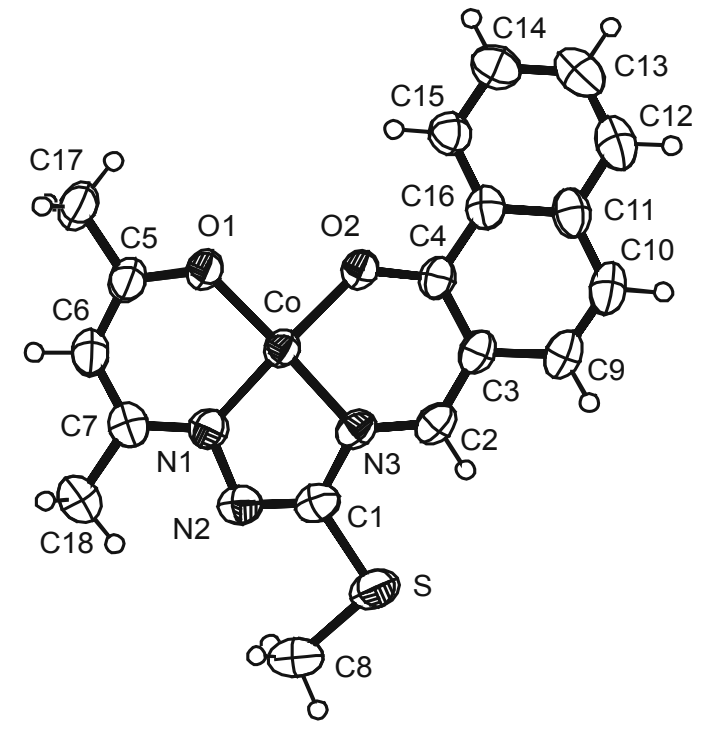

$\left[\mathrm{CoL}^{7}\right](13)$

Fig. 3. Molecular structures of the complexes $\mathbf{1 0}$ and $\mathbf{1 3}$

The above mentioned complexes 6-17 are not the only species, which are present in the system. The partial desegregation of the initial ligson produces fragments with an increased reactivity, which ensures their fit in new combinations, with an unusual arrangement of atoms and bonds. In the presence of 2,6-diformyl-4-methylphenol or oxynaphthaldehyde the formation of the complexes $\mathbf{3}$ and $\mathbf{4}$ has been also observed. These transformations prove the complexity of the process which induces the diversity of the final products. Thus, the reaction with the higher yield can not be considered as single possible transformation. It is the reaction the more advanced, but not the only process. The lockout of another process as frequently occurs by solvent implication. The interaction of benzoylacetone monoS-methylthiosemicarbazone with 2,6-diformyl-4-methylphenol in methanol gives rise to the condensation in molar ratio $1: 1$. The acetals formation prevents the condensation with the second group and thus the generation of dinuclear compound [12].

The initially prepared complexes using template methodology can be involved in the next step to build new macrocyclic edifices with a given configuration. Thus, the acetylacetone bis-(S-methylthiosemicarbazone) cobalt and nickel complexes, prepared by template reaction, were used as already assembled form, in order to produce macrocyclic systems. The macrocyclic compounds (18) and (19) [8] with cis-arrangement of thiosemicarbazidic fragments were prepared by tailoring the sodium salt of the nitromalonic dialdehyde or 1,1,3,3-tetraetoxypropane to the chelate precursor of the type 2, following the scheme 5. The compound (18) is paramagnetic, stable at high temperatures and can be thermally decomposed at $193{ }^{\circ} \mathrm{C}$. The central ion is formally in oxidation state +2 , confirmed by magnetic properties.

The macrocyclic cobalt(III) complex (19) with coordination number five can be synthesized coming from the open-chain compound $\left[\mathrm{CoL}^{1} \mathrm{I}\right] \mathrm{I} \cdot \mathrm{CH}_{3} \mathrm{OH}$ with square-pyramidal structure as matrix (or template) and 3-etoxyacroleine as tailoring agent.

The condensation of 3-etoxyacroleine with the thioamidic nitrogen atom of isothiosemicarbazidic fragment leading to the formation of a macrocyclic system was confirmed by IR spectra and mass-spectrometry. In the mass-spectra of (19), the most intense pick $[\mathrm{M}-\mathrm{I}]^{+}$and molecular pick $[\mathrm{M}]^{+}$, which belongs to cobalt complex with macrocyclic equatorial ligand were recorded. The presence of the pick $[\mathrm{M}]^{+}$, confirms the coordination of $\mathrm{I}^{-}$to the central atom. Taking into account these data square pyramidal structure with $\mathrm{I}^{-}$anion in apical position has been suggested for (19). 


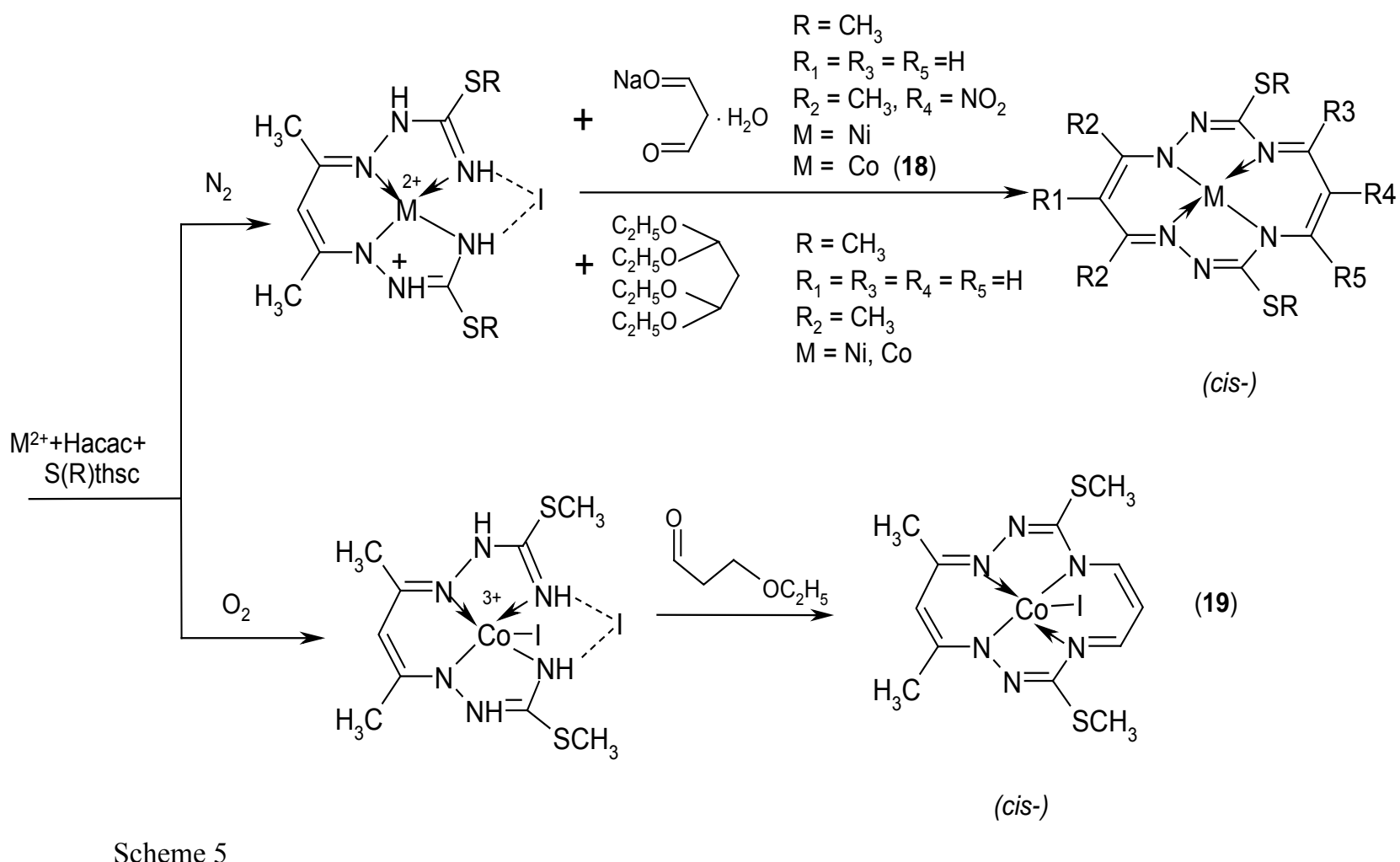

Scheme 5

Other nickel(II) and copper(II) coordination compounds $(\mathbf{2 0}$ - 23) with hexaazamacrocyclic ligands with cis-configuratrion of the isothiosemicarbazide fragments can be prepared starting from nitromalonic dialdehyde bis-(Smethylthiosemicarbazone) and 1,1,3,3-tetraetoxypropane as closing agent [8] (scheme 6):

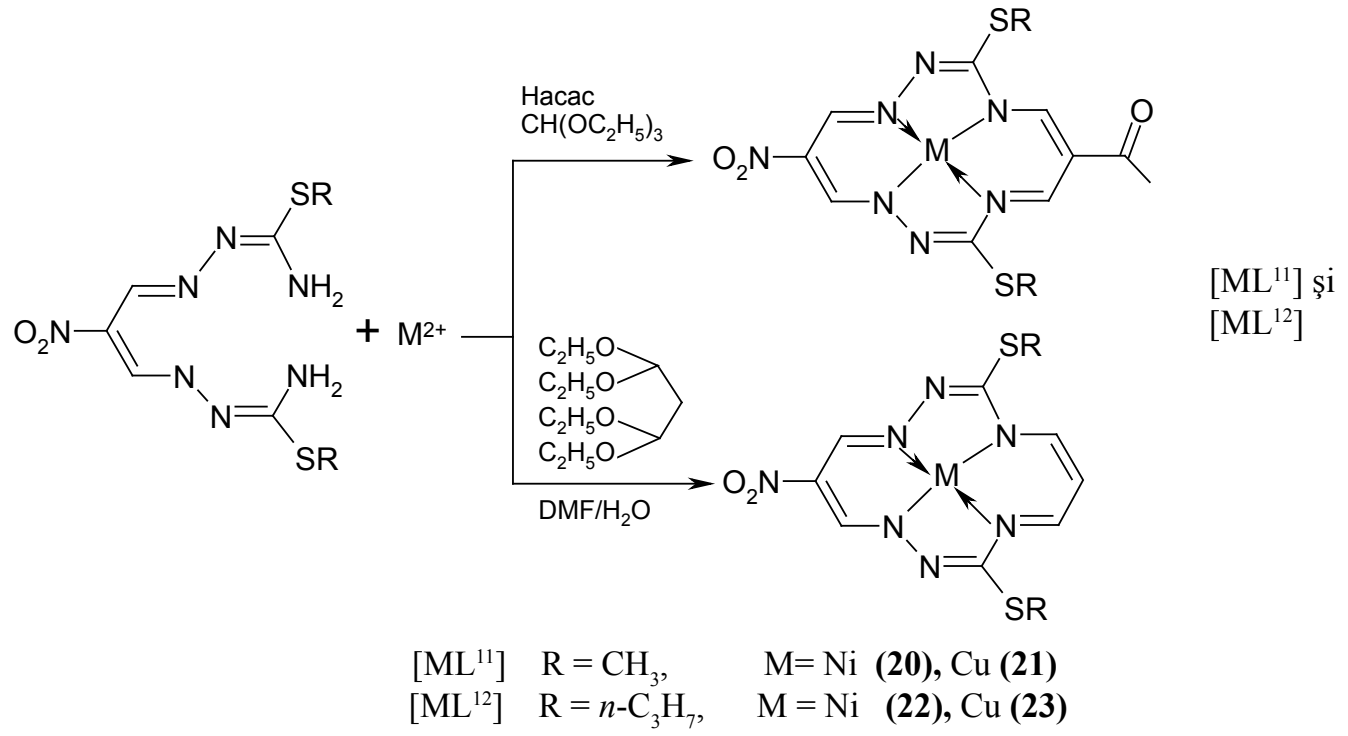

Scheme 6

Their purity, composition, structure and physico-chemical properties have been established using the elemental analysis and a number of physical methods such as, thermogravimetry, mass-spectrometry, IR, UV-VIS, NMR spectroscopy). For the copper(II) complex $\mathbf{2 3}$ an X-ray investigation was undertaken. The molecular structure of $\mathbf{2 3}$ is shown in fig. 4. 


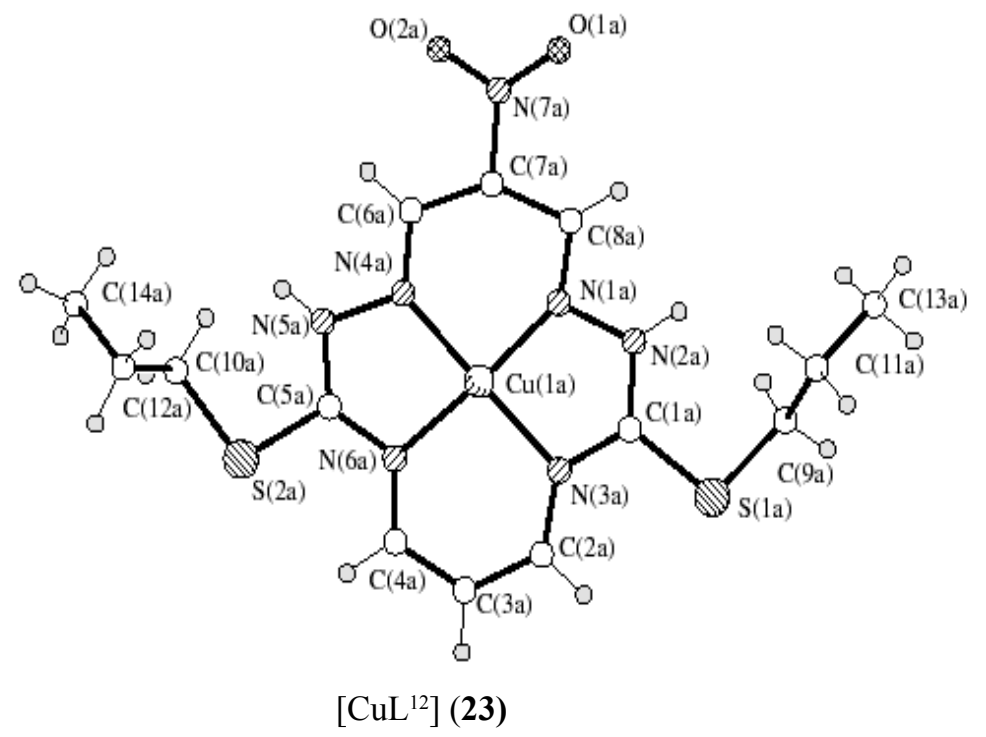

Fig. 4. Molecular structure of the complex 23

The complexes prepared using this technology has not only an original structure or a new arrangement of the atoms and bonds. They possess also a number of useful properties. So, the influence of $\mathrm{NiL}^{6}(\mathbf{8}) \mathrm{and}^{\mathrm{CuL}}{ }^{6}(\mathbf{9})$ on $^{-}$ the Aspergillus niger 412 fungus production of lipolytic enzymes were studied. The obtained result proves obvious the increasing of lipolytic activity of studied fungus, biomass yield when $\mathrm{NiL}^{6}$ and $\mathrm{CuL}^{6}$ complexes have been added.

The influence of nickel(II), copper(II) and oxovanadium(IV) cooordination compounds: $\left[\mathrm{ML}^{7}\right](\mathbf{1 0}-\mathbf{1 2})$ and $\left[\mathrm{ML}^{8}\right](14$ - 16) on pectolytic enzymes biosynthetic process by Penicillium viride fungus has been studied. Tested complexes demonstrate the stimulation properties (the biomass accumulation, the increase of enzymatic activity). These properties are influenced by the presence of $\mathrm{Ni}^{2+}$ and $\mathrm{Cu}^{2+}$ ions and ligands nature [14].

Some complexes of nickel(II) $\left[\mathrm{NiL}^{7}\right](\mathbf{1 0})$ and $\left[\mathrm{NiL}^{8}\right](\mathbf{1 4})$ have been studied as dying agents for plastic $[15$, 16]. Varying the concentration of coloring agent and polymer type has been obtained the samples with different intensity and transparency of the color. These compounds are suitable for coloring the plastic (polyethylene and polystyrene) with high photostability.

\section{Conclusions}

The geometry, composition and the properties of compounds prepared by template methodology using the dicarbonylic blocks and alkylisothiosemicarbazidic fragments can be diversified by the variation of the carbonylic component, template center, and substituents. The nature of the used ß-dicarbonylic blocks for assembling, as well as the structure of the precursors are responsible for the succession of the condensation of the reactant blocks. By their selection can be targeted the synthesis of coordination compounds with trans-and cis-arrangement of isothiosemicarbazidic fragments. Some compounds of this type display biologic activity and stimulate the biosynthesis process of some fungus - producers of extracellular hydrolyze (biomass accumulation, the increase of enzymatic activity). The biologic activity is influenced by the nature of metal ion and of ligands. The colouristic properties of some complexes can be used to colouring the plastics [15].

\section{Acknowledgements}

The authors gratefully acknowledge MRDA/CRDF grant (MTFP-1018A Follow on).

\section{References}

[1] Revenco, M. D. Ph. D. Thesis, Odessa, 1991.

[2] Arion, V.; Revenco, M.; Gradinaru, J. Reviews in Inorg. Chem., 2001, p. 1.

[3] Gerbeleu, N. V.; Arion, V.B.; Burges, J. Template synthesis of macrocyclic compound, 1999, Wiley-VCH: Weinheim, $565 \mathrm{p}$.

[4] Beraldo, H.; Gambino, D. Mini. Rev. Med. Chem., 4, 2004, p. 159.

[5] Leovac, V. M.; Cesljevic, V. I. Coordination Chemistry of Isothiosemicarbazide and their Derivatives, Department of Chemistry, Faculty of Sciences, Novi Sad, 2002.

[6] Arion, V. B. Ph.D. Thesis, Kiev, 1985.

[7] Gradinaru, J. I. Ph.D. Thesis. Institute of Chemistry of ASM, 1993. 
[8] Leovac, V. M.; Bogdanovic, G. A.; Cesljevic, V. et al. Acta Cryst., Sect. C., 2000, 56, pp. 936-938.

[9] Gradinaru, D. I.; Gerbeleu, N. V.; Arion, V. B.; Simonov, Yu. A.; Popovich, M. A. and Bourosh, P.N. Russian Journal of Inorganic Chemistry, 2000, 45, pp. 533-543.

[10] Gradinaru, J. I.; Simonov, Yu. A.; Arion, V. B.; Bourosh, P. N.; Popovici, M. A.; Gerbeleu, N. V. Inorganica Chimica Acta, 2001, 313, pp. 30-36.

[11] Gradinaru, J.; Malinovskii, S.; Popovici, M.; Gdaniec, M. Krystallografia (Russian), 2005, 50, pp. $217-224$.

[12] Gradinaru, J. I.; Forni, A.; Simonov, Yu. A.; Popovici, M. A.; Zecchin, S.; Gdaniec, M.; Fenton, D. Inorganica Chimica Acta, 2004, 357, pp. 2728-2736.

[13] Cocu, M. A.; Tiurina, J. P.; Gradinaru, J. I.; Deseatnic, A. A.; Gerbeleu, N. V.; Sirbu, T.F. The XXII ${ }^{\text {th }}$ International Chiugaev Conference Reading in memory of Academician A.B. Ablov. June 20/24, 2005, Chisinau, Moldova, p. 387.

[14] Cocu, M. A.; Clapco, S.; Gărbălău, N. V.; Grădinaru, J. I.; Deseatnic, A. A. Romanian International Conference on Chemistry and Chemical Engineering- RICCCE XIV. Bucharest, Romania, September $22^{\text {nd }}-24^{\text {th }}, 2005$, p. S01$51-\mathrm{S} 01-53$.

[15] Cocu, M.; Gărbălău, N.; Manole, Ş.; Grădinaru, J.; Forni, A. Patent MD 2881 G2 2005.

[16] Cocu, M.; Gărbălău, N.; Manole, Ş.; Grădinaru, J.; Forni, A. Patent MD 3431 G2 2007. 